B. GROUND-BASED OBSERVATIONS OF

SPECTRA RELEVANT TO THE ULTRAVIOLET 


\title{
REVIEW OF GROUND-BASED OBSERVATIONS OF SPECTRA RELEVANT TO THE ULTRAVIOLET
}

\author{
M. W. FEAST \\ Radcliffe Observatory, Pretoria, South Africa
}

\section{Introduction}

Up to the present the chief results of investigations of stellar line spectra in the extreme ultraviolet have been limited to two fairly distinct fields. In the case of the Sun the data have referred mainly to the chromosphere and also to the region of the temperature minimum at the top of the photosphere and to the corona. We may hope that observations will eventually be extended to the chromospheric ultraviolet spectra of other stars. In the case of the early type stars the most spectacular results so far obtained have concerned the evidence for mass loss by several of these stars. I have therefore chosen to spend most of the available time discussing some of the ways of studying from the ground, first the chromospheres of stars other than the Sun and secondly mass loss from stars.

\section{Observations Relating to Stellar Chromospheres}

\section{A. Ca II H AND K EMISSION IN STARS}

Probably the most comprehensive attack on the problem of stellar chromospheres has been $\mathrm{O}$. $\mathrm{C}$. Wilson's work on $\mathrm{H}$ and $\mathrm{K}$ emission in late type stars $[1,2,3,4,5]$. By analogy with the Sun it has been easy to suppose that the emission arises at the chromospheric level and that in many late type stars the degree of chromospheric activity is much greater than in the Sun. The main conclusions of Wilson's work are well known. There is a linear relation, independent of spectral type, extending over about 15 magnitudes between the $\mathrm{K}_{2}$ emission line widths and the visual absolute magnitude (the Wilson-Bappu effect). For stars near the main sequence there is evidence that the intensity of Ca II emission decreases with age. This evidence comes both from intercomparing clusters of different ages and from stars in the general field whose relative ages can be fixed by the Stromgren-Perry photometry. Wilson also finds that chromospheric $\mathrm{H}$ and $\mathrm{K}$ generally become inconspicuous earlier than about $\mathrm{F} 4$ on the main sequence. This is just the point where rotational velocities of main sequence stars increase sharply and where theory predicts that main sequence stars should cease to have hydrogen convection zones. These results suggest therefore that the formation of a chromosphere in a normal star depends on the existence of a hydrogen convection zone. In addition stars with hydrogen convection zones are assumed by analogy with the Sun to have stellar winds. The interaction of these winds with stellar magnetic fields leads to rotational deceleration. This aspect of the problem has been carried a step further by Kraft [6] who finds evidence from a detailed investigation of the rota- 
tional velocities of solar type stars, that such stars are indeed rotationally decelerated after reaching the main sequence.

Further statistical work on $\mathrm{H}$ and $\mathrm{K}$ emission in late type stars should benefit considerably from the publication by Warner [7] of emission line strengths and widths in 200 southern $\mathrm{G}, \mathrm{K}$ and $\mathrm{M}$ type stars.

Wilson [8] is engaged in a programme of accurate intensity measurements of $\mathrm{H}$ and $\mathrm{K}$ emission which is inspired by the desire to find stellar equivalents to the solar sunspot cycle. So far no significant variations have been found in the total flux determined photoelectrically. However changes (particularly changes in profile) of the $\mathrm{K}_{232}$ structure have been found by a number of workers including Griffin [9], Deutsch [10] and Liller [11]. Liller found evidence for variations in a number of $G$ and $K$ type stars. For Aldebaran ( $\alpha$ Tau, K5 III) he found, in addition to general variability, a flare-like increase in the intensity of the violet component of $K_{2}$. The increase was about $19 \%$ and lasted half an hour. Deutsch, whose work extends over a long period, finds evidence for variable $H$ and $K$ profiles in a number of $K$ and $M$ giants as well as a good deal of fine structure in the lines. He has also looked for possible periodicities in the phenomena (this volume, pp. 199-208).

Not all stars showing CaII emission obey the Wilson-Bappu effect. The transient CaII emission lines in cepheids are considerably wider than would be predicted [12] (for example in X Cyg, $213 \mathrm{~km} \mathrm{sec}^{-1}$ against an expected $140 \mathrm{~km} \mathrm{sec}^{-1}$ ). Perhaps associated with this is the fact that the $\mathrm{K}_{3}$ reversal is much stronger in cepheids than in non-variable stars, probably indicating that the emission comes from an abnormally deep layer [13]. It will be recalled that there is good evidence that the complex emission spectrum found in Mira variables which can include $\mathrm{H}$ and $\mathrm{K}$, arises at ather deep level in the atmosphere. One might hesitate to call this spectrum chromospheric but it may well be basically a closely related phenomenon being associated with energy derived from the hydrogen convection zone. Because the basic mechanism of the $\mathrm{H}$ and $\mathrm{K}$ widths in stellar spectra remains somewhat obscure, progress may as well come from detailed studies of abnormal cases such as the cepheids or the Mira variables as from normal stars.

Whilst chromospheric $\mathrm{H}$ and $\mathrm{K}$ are, apparently, generally missing from early type stars a couple of interesting exceptions have been found by Warner [14, 15].* Canopus (F0 Ib) shows emission but this is much too narrow $\left(50 \mathrm{~km} \mathrm{sec}^{-1}\right.$ against a predicted $150 \mathrm{~km} \mathrm{sec}^{-1}$ for the Wilson-Bappu relationship. A particularly interesting case is the well known binary $\gamma$ Vir with its two equal F0 V components. The following points are worth noticing. (1) Emission is found in $\gamma$ Vir $\mathrm{N}$ with a width of $43 \mathrm{~km} \mathrm{sec}^{-1}$ agreeing with the Wilson-Bappu relation. ${ }^{\dagger}(2)$ Since $\gamma$ Vir $\mathrm{S}$ does not show emission there can

\footnotetext{
* See also the case of $\gamma$ Boo (A7 III) mentioned by Mrs Praderie (this volume, p. 69).

+ Warner notes that $\gamma$ Vir $\mathrm{N}$ has a significant amount of rotation $\left(v \sin i \sim 16 \mathrm{~km} \mathrm{sec}^{-1}\right)$. This does not appear to be at all abnormal. Stars lower down the main sequence with $v \sin i \sim 35 \mathrm{~km} \mathrm{sec}^{-1}$ and strong $\mathrm{H}$ and $\mathrm{K}$ emission are known [5,6]. In fact Kraft's principal conclusion was "for stars less massive than $M / M_{\odot}=1.25$ the largest rotational velocities are associated with stars having active chromospheres".
} 
apparently be a significant range of emission intensities in stars of the same mass and age. (3) $\gamma$ Vir $\mathrm{N}$ has a magnetic field of -390 gauss [16] suggesting a connection between high magnetic fields and high chromospheric activity. (4) There is evidence for (abnormal?) surface nuclear activity in these stars. Zirin [17] finds evidence from HeI $10830 \AA$ for both $\mathrm{He}^{3}$ and $\mathrm{He}^{4}$ in $\gamma$ Vir $\mathrm{N}$ (though mass motions in the chromosphere could falsify this conclusion). Both stars have extreme overabundances of lithium (by a factor of about 100) [18]. The beryllium abundance on the other hand is low and the $\mathrm{Li} / \mathrm{Be}$ ratio is consequently extremely high [19], about 640 in $\gamma$ Vir $\mathrm{N}$ and half this in $\gamma$ Vir $\mathrm{S}$. This together with the low $\mathrm{Li}^{6} / \mathrm{Li}^{7}$ ratio in $\gamma$ Vir $\mathrm{N}$ leads Conti to suggest that spallation has involved particles with energies of tens of $\mathrm{MeV}$ rather than the hundreds of $\mathrm{MeV}$ that appear necessary for spallation reactions in solar type stars. Work of this type gives hope that it will eventually be possible to draw together work on stellar chromospheres, stellar magnetic fields and nuclear reactions in stellar atmospheres; fields between which only vague relationships at present appear to exist.

\section{B. OTHER CHROMOSPHERIC EMISSION LINES}

There are a number of other ways of investigating the outer regions of stars from studies of emission lines, none of which have as yet been extensively exploited. Wilson [20] has shown that $\mathrm{H} \varepsilon$ emission occurs in many late type stars strengthening in general towards the later types. It seems most likely that this is chromospheric, $\mathrm{H} \varepsilon$ being most easily seen because it falls on the wing of the strong photospheric Ca II absorption.

FeII emission lines are found in a considerable number of peculiar objects. However Herzberg [21] showed that permitted multiplets near $3200 \AA$ occurred in emission in the normal M type supergiants $\alpha$ Her and $\alpha$ Sco, and more recently Bidelman [22] has noted them as a normal feature in $M$ giants and supergiants. They also occur in $\mathrm{dMe}$ stars [23]. It seems most likely that these lines are chromospheric in origin and a quantitative study of them could be very valuable. For instance little is known about the line widths and shapes. Herzberg's reproductions suggest that the Fe II lines in $\alpha$ Her and $\alpha$ Sco are significantly narrower than the $\mathrm{H}$ and $\mathrm{K}$ emission lines which are about $150 \mathrm{~km} \mathrm{sec}^{-1}$ wide in these stars. In $\alpha$ Ori, Weymann [24] made the significant discovery that the stronger of these Fe II emission lines is self reversed. Even so the widths appear to be significantly less than the $\mathrm{H}$ and $\mathrm{K}$ widths (roughly $149 \mathrm{~km} \mathrm{sec}^{-1}$ as against $186 \mathrm{~km} \mathrm{sec}^{-1}$ ) whilst the weaker Fe II lines are not self reversed and are apparently somewhat narrower. There is evidently a strong indication here that (contrary to what has sometimes been supposed) the Wilson-Bappu effect cannot be interpreted as due to turbulent broadening in an optically thin model. Swings and Swings $[25,25 \mathrm{a}]$ have recently drawn attention to the importance of measurements of the relative intensities of these $\sim 3200 \AA$ Fe II lines for studies of the level of excitation in the emitting region. In quantitative work considerable care will probably be necessary in allowing for the effects of the superimposed photospheric spectrum and for any overlying absorption. 


\section{CHROMOSPHERIC ABSORPTION}

Whilst chromospheric effects may be found in a considerable number of absorption lines there are one or two chromospheric absorption line problems which are of particular importance. Evidence from the Sun suggests that the cores of $\mathrm{H} \alpha$ absorption in late type stars are of chromospheric origin. In accord with this interpretation these cores show an increasing width with increasing luminosity, an absorption WilsonBappu effect [26]. An example of what may be achieved from detailed studies of $\mathrm{H} \alpha$, at least in the case of a variable star, has been given recently by Rodgers and Bell [27]. In the long period (35.5 days) cepheid $l$ Carinae they found evidence for multiple absorption cores. One of the components is observed throughout the cycle and has a velocity close to the $\gamma$-velocity of the star. They concluded that there is a chromospheric shell which does not take part in the cepheid pulsation.

In the Sun HeI $10830 \AA$ absorption is known to be wholly chromospheric. Vaughan and Zirin [28] have studied this line in late type stars. Because of its high excitation potential the presence of the line gives evidence for a hot chromosphere $(T>20000 \mathrm{~K})$. The line is found mainly in $\mathrm{G}$ and $\mathrm{K}$ type stars and its strength correlates roughly with $\mathrm{K}_{2}$ emission intensity. There is also a luminosity effect, the absorption being weaker in the dwarfs. Evidence of temporal variations in the line intensity has been found and macroscopic motions in the absorbing region are indicated by velocity shifts which are generally negative (i.e. rising) and up to $50 \mathrm{~km} \mathrm{sec}^{-1}$. The line is generally fairly diffuse $(\sim 1.5 \AA$ wide) but with no correlation of width and luminosity (no Wilson-Bappu effect).

The occurrence of $\mathrm{HeI}_{3}(5876 \AA)$ in absorption in a few late type stars has been reported [29]. Further work on this line is very desirable though care has to be taken about some faint terrestrial lines in this region of the spectrum. Rather surprisingly Wilson and Aly find in the three stars they measured that the line is displaced longward (by about $0.3 \AA$ ) in contrast to the general shortward displacement of $10830 \AA$.

\section{THE CHROMOSPHERES OF THE R CORONAE BOREALIS STARS}

Amongst the various peculiar stars the $\mathrm{R} \mathrm{CrB}$ variables seem worth mentioning here because they hold out prospects for studies of the outer atmospheres of stars which would probably not be otherwise possible. The two best studied stars of this class R CrB itself and RY Sgr are similar carbon rich, hydrogen poor, F-type supergiants which at irregular intervals undergo large and rapid decreases in light. At such times the stars show a very rich emission spectrum mostly of ionized metals (some 350 emission lines have been measured at medium dispersion during the recent minimum of RY Sgr). There is a sharp drop in continuum intensity, possibly due to a large increase in opacity and this allows the observation of emission from the chromosphere or upper photosphere. In RY Sgr an emission continuum has been observed which has been attributed to the electron attachment spectrum of $\mathrm{CN}$ [30]. Marked changes take place in the emission spectrum and we are apparently observing the decay of chromospheric emission when its source of excitation is cut off. Two further points about 
R CrB stars are relevant to our general topic. Firstly, He I $5876 \AA$ absorption has been identified in their spectra near maximum light. In view of the almost certain chromospheric origin of this line in a number of normal late type stars it is clearly dangerous to attempt a determination of helium abundance from the line. Secondly, simultaneous UBV photometry and spectroscopy shows that there are times when RY Sgr has a normal spectrum (i.e. as at maximum) but is faint and reddened. This is good evidence for the formation of an absorbing cloud of particles well clear of the stellar surface. Since this presents a unique opportunity to study variable circumstellar reddening in detail, attempts are expected to extend the photometry into the extreme ultra-violet. However caution should be exercised in interpreting any results that are obtained since chromospheric emission in the ultraviolet could well complicate the picture.

\section{E. OTHER DATA}

I am aware of having omitted a number of important aspects of the chromospheric problem. In particular I have no time to discuss the study of eclipsing systems which reveal the complex mass motions in the chromospheres of the $\zeta$ Aurigae type supergiants (cf. [31]).

\section{Mass Loss from Stars}

The other chief topic where there is much in common between ultraviolet and groundbased observations is that of mass loss. Here I shall be very restrictive, leaving out entirely the work of Deutsch and others on mass loss in late type stars. It has long been known that mass loss takes place from the atmospheres of some early type stars. There is an extensive literature on WR stars, P Cygni stars, Be stars etc. much of which is directly relevant to the present topic. However there are excellent recent general reviews of these fields available (for instance by Underhill [32, 33, 34] and I have chosen simply to discuss the most recent work in a little detail.

\section{A. THE OB SUPERGIANTS}

The work of the last few years which has seemed most closely related to the ultraviolet work on $\mathrm{OB}$ stars has been that of Hutchings $[35,36]$. From studies of velocities of lines arising at different mean levels he has been able to deduce the existence of extended atmospheres for some early type supergiants in which matter is being accelerated outwards. Velocities up to $600 \mathrm{~km} \mathrm{sec}^{-1}$ were found in the extreme outer parts of the stars. These are somewhat smaller than the velocities found in the ultraviolet observations but it has seemed that the various observations might fit together quite well.

A number of points arise which link this work with more general questions. It is reasonable to ask if the large amount of low and medium dispersion work on OB stars can tell us whether mass loss, observable from the ground, is a common occurrence. Hutchings studied the very luminous B1 Ia-O star $\zeta^{1}$ Sco. For this star a normal radial velocity measurer would probably omit the lower Balmer lines which have $\mathrm{P}$ Cygni 
profiles but would almost certainly use many lines (HeI, MgII, OII, NiI, etc.) for which substantial velocity shifts are found by Hutchings. Probably one would expect the measured velocity to be algebraically smaller than the true stellar velocity by $10 \mathrm{~km} \mathrm{sec}^{-1}$ or so in this case. A direct comparison is not possible because the velocity of the star is variable, presumably due to the varying velocity fields in the atmosphere. Nevertheless if net outward velocities were present in the atmospheres of a substantial number of $O B$ stars we should expect kinematical investigations to show significant negative $\mathrm{K}$ terms. Fairly extensive investigations of this type have in fact been carried out [37], dividing the stars according to type and luminosity class. No significant $\mathrm{K}$ terms were found except for a positive $\mathrm{K}$ term for O-type stars which is probably at least partly due to a gravitational red shift. If anything most $\mathrm{K}$ terms are slightly positive. This suggests that so far as ground-based observations are concerned, evidence for mass loss on the scale of that found for $\zeta^{1}$ Sco will not be found for any large number of $\mathrm{OB}$ stars (including supergiants). This conclusion would however not hold if for some reason the stars showing mass loss effects were just the ones omitted in kinematical investigations because they showed variable radial velocities. Furthermore these results do not preclude the general occurrence of mass loss amongst supergiants of the very highest luminosity which probably form a small fraction of the total number of supergiants in the analysis. One might expect that radial velocities of early type stars in galactic clusters would throw some light on the mass loss problem. In fact it has been known for some years $[38,39]$ that early-type supergiants in clusters tend to show greater positive velocities than the giants. This effect is not yet understood but it may as well be due to some peculiar effect in the giants as in the supergiants.

The problem of $\mathrm{H} \alpha$ emission in early type supergiants is intimately connected with the problem of mass loss. Andrews [40] has shown that the absorption strength (measured photoelectrically) is a good luminosity indicator except amongst main sequence Be stars where the emission destroys the correlation. However $\mathrm{H} \alpha$ strength remains well correlated with luminosity amongst the supergiants even when the line is going over into emission. So the occurrence and strength of the emission is closely connected with luminosity. Similar conclusions were deduced by Abt [41, 42]. These results appear to fit naturally with the spectroscopic work on the early type supergiant members of the Magellanic Clouds. Here the emission line stars at a given spectral type are strongly concentrated amongst the most luminous stars [43]. Many of these stars show P Cygni effects and the Magellanic Clouds results show rather clearly that instability and consequent mass loss increases as one goes towards the supergiants of the highest luminosity. Apparently these effects are to be attributed to the increasing importance of radiation pressure in these atmospheres.

The mere presence of Balmer emission in a supergiant is apparently no guarantee of mass loss. Peterson [44] has recently found that one can predict from a non-LTE theory that bright early-type supergiants may show (double) $\mathrm{H} \alpha$ emission even under conditions of hydrostatic equilibrium. Of course both in the Galaxy and the Magellanic Clouds, the majority of the very luminous stars we are discussing show definite evidence of mass loss from P Cygni profiles. Nevertheless the effects predicted by Peter- 
son may need taking into account in detailed interpretations of the $\mathrm{H} \alpha$ profiles of these stars.

Thus various ground-based observations give evidence for the general occurrence of mass loss amongst the most luminous early-type supergiants. There is as yet however little ground-based evidence for mass loss amongst somewhat fainter supergiants, some of which are shown by the ultraviolet observations to be ejecting matter (e.g. [45]).

The discussion of this section will require substantial modification if a recent suggestion by Underhill [46] receives confirmation. She has suggested that the early-type Ia supergiants have relatively low masses and large underabundances of hydrogen. If this is indeed the case these stars may no longer be regarded as simply an extension of the Ib supergiants to greater masses. They would apparently be in a very advanced evolutionary stage.

\section{B. THE Of STARS}

The Of stars are a little understood group of stars which are of particular interest at this symposium since high velocity mass ejection has been established for the Of star $\zeta$ Pup from ultraviolet work. Hutchings [35] has recently discussed the marked mass ejection effects in HD 152408. The high negative radial velocity of this star was interpreted long ago by Struve [47] as indicating a rapidly expanding shell. Spectroscopic workers have generally hesitated to regard the star as a normal Of star and Struve remarked that it might be classed as a hot P Cygni star. HD 151804 which was also studied by Hutchings appears a more normal Of star and the atmospheric velocity effects though present are less pronounced. In this case low dispersion work might indicate a velocity of 20 or $30 \mathrm{~km} \mathrm{sec}^{-1}$ lower than the true stellar velocity.

It is of importance both for problems of mass loss and for problems of galactic kinematics to know whether the measured radial velocities of a substantial number of Of stars are falsified by atmospheric effects. This can be seen from Blaauw's discussion of the run-away stars [48]. Of the 5 stars of type $\mathrm{O} 8$ or earlier in his discussion, 3 are of type Of and one of these is HD 152408 which must be rejected in the light of the work of Struve and Hutchings. In Table I are listed the residual velocities (i.e. velocities corrected for solar motion and galactic rotation) for 20 galactic Of stars with published radial velocities and photometry. Distances were calculated using an absolute magnitude of -7.0 (see below) and the residuals were calculated using the $\omega(R)$ results for OB stars and interstellar gas [37]. Six of the 20 stars have residuals greater than $35 \mathrm{~km} \mathrm{sec}^{-1}$. That is, they are run-away stars if the measured velocities reflect the true stellar velocities (notice that HD 152408 is not included in the list). Four of the 6 high velocity stars have negative, and two positive, residuals. The existence of some positive residuals presumably indicates that some Of stars are real run-away objects. $+60^{\circ} 2522$ is only marginally in this category but HD 157857 is clearly in this class. Of the stars with large negative residuals one is the spectroscopic binary $29 \mathrm{CMa}$ for which the emission lines give a much more positive $\gamma$-velocity (by $66 \mathrm{~km} \mathrm{sec}^{-1}$ ) than the absorption lines which were used for Table I [49]. The quoted 


\section{TABLE I}

Of stars

\begin{tabular}{llcl} 
& & & \\
HD & $r(\mathrm{kpc})$ & $\begin{array}{l}\text { Residual velocity } \\
\left(\mathrm{km} \mathrm{sec}^{-1}\right)\end{array}$ \\
\hline 16691 & 4.6 & +9 & \\
14947 & 3.3 & -13 & \\
15570 & 2.5 & +9 & \\
108 & 4.0 & -10 & \\
57060 & 2.0 & -51 & $29 \mathrm{CMa}$ \\
66811 & 0.7 & -69 & $\zeta$ Pup \\
157857 & 2.9 & +55 & \\
166734 & 1.7 & -2 & \\
167971 & 1.8 & +9 & \\
171589 & 5.2 & -14 & \\
175754 & 4.8 & -31 & \\
188001 & 2.6 & -1 & 9 Sge \\
192281 & 3.0 & -50 & \\
192639 & 2.8 & +6 & \\
193514 & 2.6 & -11 & \\
210839 & 1.2 & -53 & $\lambda$ Cep \\
225160 & 4.8 & +16 & \\
$+60^{\circ} 2522$ & 5.0 & +37 & \\
$190429 \mathrm{br}$ & 3.5 & -11 & \\
148937 & 2.1 & -29 &
\end{tabular}

velocity is thus very suspect. However it does not follow that all the negative velocities must be rejected as probably badly affected by mass ejection.

A few radial velocities are available for stars in IC 1805 including an Of member. The velocities do not appear to be of high accuracy but they indicate that if anything the velocity of the Of star is somewhat positive with respect to the other stars. There is evidently need for detailed work on as many Of stars as possible to determine the influence of mass ejection effects on the measured velocities.

The 7th magnitude Of star HD 148937 is of considerable interest. As Henize [50] and Gum [51] noticed the star is centrally located between the two nebulosities NGC 6164-5. The object was at one time classed with the planetary nebulae and would in that case be very close $(\sim 150 \mathrm{psc})$ [50]. However the star has the colour of a normal reddened $O$ type star [52] and strong interstellar lines as well as a low proper motion, whilst the nebulosity is of low excitation: It appears now to be agreed $[52,53]$ that the star must be considered a normal, high luminosity, Of type. It is of considerable interest to find an Of star associated with nebulosity which gives the impression (see photographs in [52] and [54]) of having been ejected from the star. A full study of the kinematics of the nebulosity is clearly desirable.*

* In presenting this paper at Lunteren it was pointed out that the southern nebulosity had a radial velocity close to the published [55] high negative radial velocity of the star. This suggested that the system might have a high space motion. Subsequent work has confirmed this approximate agreement but has shown that the northern nebulosity has a much more positive velocity. Evidently the stellar velocity is affected by mass loss effects. 
It might be supposed that, as in the case of the OB supergiants, the mass ejection from Of stars was the result of radiation pressure and this view has been taken by some workers (compare Hutchings and Solomon, this volume, pp. 209-214; 236-240). However at least two other effects may be suggested for the instability of these stars.

(1) It has been suggested that the Of stars are somewhat evolved massive stars and are related to main sequence $O$ stars in the same way that the early-type Be stars are related to the main sequence $O B$ stars $[32,56]$. The early-type Be stars appear to be slightly evolved objects and to show the effects of rotational instability in evolving stars (e.g. [57, 58, 59]).

(2) The absolute magnitudes of the galactic Of stars have been discussed by a number of workers, Kopilov, Hack, Underhill and others. Whilst there remains some doubt as to the spread of luminosities amongst these objects there seems little doubt that some of them are highly luminous, $M_{V}$ up to about -7 . For instance the Of stars in the I Sco association have an absolute magnitude near $-7 .^{*}$ Van den Bergh [56] quotes a value of -6.6 for the Of star in IC 1805 whilst the two known Of stars in the Magellanic Clouds [43] also have absolute magnitudes of about -7 . If the Of stars are near main-sequence objects then these high luminosities indicate such high masses (of the order of $100 M_{\odot}$ ) that the stars would be expected to show the effects of pulsational instability which are predicted to set the upper limit to masses on the main sequence $[61,62]$. It may also be noted here that the agreement between the absolute magnitudes of the Of stars in the Galaxy and the Magellanic Clouds suggests that it is unnecessary to attempt to explain away the high luminosities of the Magellanic Cloud objects by regarding them as unresolved groups of stars [61].

Further work appears to be necessary to decide on the relative importances of rotational instability, pulsational instability and atmospheric radiation pressure in the case of the Of stars. In this type of theorizing however it is sobering to recall that Wilson [63] showed long ago that the nucleus of the planetary nebula NGC 2392 which is similar to an Of star but is presumed to be a sub-luminous object has an expanding atmosphere with outward acceleration closely similar to that found by Hutchings in the highly luminous Of's. It is not at all clear whether we have a number of different mechanisms producing similar observational effects or whether there is one mechanism working over a large range of stellar masses and luminosities.

\section{RAPID CHANGES IN THE SPECTRA OF THE EARLY-TYPE STARS}

Although mass loss is established for a number of early-type stars we know, as yet, little about the variations of the mass loss with time. Changes in radial velocity and line shape, especially at $\mathrm{H} \alpha$, are known for several bright supergiants. More recently evidence has been obtained for quite rapid changes in the spectra of some early type stars (cf. $[64,65,66])$. Some of these effects such as variations within a few minutes of the emission structure of $\mathbf{P}$ Cygni seem directly connected with the mass loss problem.

* Absolute magnitudes about 1 magnitude fainter have recently been quoted for these stars [60] but this appears to be due to a numerical error. 
A particularly interesting problem concerns the Of stars. Already some years ago Oke found evidence for variations in the intensities of the emission lines in Of stars in the course of a few hours [67]. It would be important to follow up this work in more detail since periods of about this length would be expected if the basic cause of the of phenomenon is pulsational instability.

\section{Ground-Based Predictions of Ultraviolet Spectra}

To conclude I should mention briefly a quite different topic. There are a number of cases in which ground-based observations have been used to make definite predictions of ultraviolet spectra. One thinks particularly of Zanstra's work predicting ultraviolet continua of central stars of planetaries from the observed nebular line emission. Similarly Bowen's fluorescence mechanism to explain mutilated multiplets in nebulae requires strong He II $304 \AA$ and other emission lines in this region. Direct confirmation of these predictions may be difficult or impossible because of interstellar absorption. However a more tractable case is that of the Mira variables. The application of Bowen's fluorescence mechanism to the observed emission spectra of these stars by Thackeray [68] leads to the prediction of a number of strong ultraviolet emission lines in these stars particularly the $\mathrm{Mg}$ II doublet at $2800 \AA$. It is even possible $[69,70]$ to predict from the observed emission lines that the $\mathrm{Mg}_{\text {II }}$ lines will be self reversed with the short wavelength emission wing strongest. One supposes that these ultraviolet lines must be abnormally intense in Mira variables. Direct observation of these lines is desirable not only to verify the predictions but to help understand the basic cause of line emission in Mira variables.

\section{Acknowledgements}

I am indebted to A. D. Thackeray and T. Lloyd Evans who kindly read and commented on an early draft of this paper.

\section{References}

[1] Wilson, O. C. and Bappu, M. K. V.: 1957, Astrophys. J. 125, 661.

[2] Wilson, O. C.: 1959, Astrophys. J. 130, 499.

[3] Wilson, O. C.: 1963, Astrophys. J. 138, 832.

[4] Wilson, O. C. and Skumanich, A.: 1964, Astrophys. J. 140, 1401.

[5] Wilson, O. C.: 1966, Astrophys. J. 144, 695.

[6] Kraft, R. P.: 1967, Astrophys. J. 150, 551.

[7] Warner, B.: 1969, Monthly Notices Roy. Astron. Soc. 144, 333.

[8] Wilson, O. C.: 1968, Astrophys. J. 153, 221.

[9] Griffin, R. F.: 1963, Observatory 83, 255.

[10] Deutsch, A. J.: 1967, Publ. Astron. Soc. Pacific 79, 431.

[11] Liller, W.: 1968, Astrophys. J. 151, 589.

[12] Kraft, R. P.: 1960, in Stars and Stellar Systems, Vol. VI (ed. by J. L. Greenstein), University of Chicago Press, Chicago.

[13] Herbig, G. H.: 1952, Astrophys. J. 116, 369.

[14] Warner, B.: 1966, Observatory 86, 82. 
[15] Warner, B.: 1968, Observatory 88, 217.

[16] Babcock, H. W.: 1958, Astrophys. J. Suppl.Ser. 3, 141.

[17] Zirin, H.: Astrophys. J. 152, L177.

[18] Conti, P. S. and Danziger, I. J.: 1964, Astrophys. J. 146, 383.

[19] Conti, P. S.: 1969, Astrophys. J. 155, L167.

[20] Wilson, O.C.: 1957, Astrophys. J. 126, 46.

[21] Herzberg, G.: 1948, Astrophys. J. 107, 94.

[22] Bidelman, W. P.: 1961, Astron. J. 66, 453; 1961 Trans. I. A.U. XIA, 305.

[23] Wildt, R.: 1951, Astron.J.56, 51.

[24] Weymann, R.: 1962, Astrophys. J. 136, 844.

[25] Swings, J. P. and Swings, P.: 1967, Astrophys. Letters 1, 54.

[25a] Swings, J. P.: 1969, Astrophys. J. 155, 515.

[26] Kraft, R. P., Preston, G. W., and Wolff, S. C.: 1964, Astrophys. J. 140, 235.

[27] Rodgers, A. W. and Bell, R. A.: 1968, Monthly Notices Roy. Astron. Soc. 138, 23.

[28] Vaughan, A. H. and Zirin, H.: 1956, Astrophys. J. 152, 123.

[29] Wilson, O. C. and Aly, M. K.: 1968, Publ. Astron. Soc. Pacific 68, 149.

[30] Feast, M. W.: 1969, Non-Periodic Phenomena in Variable Stars, (ed. by L. Detre), Academic Press, Budapest p. 253.

[31] Wilson, O. C.: 1960, in Stars and Stellar Systems, Vol. VI: Stellar Atmospheres (ed. by J. L. Greenstein), University of Chicago Press, Chicago.

[32] Underhill, A. B.: 1966, The Early-Type Stars, Reidel, Dordrecht, The Netherlands.

[33] Underhill, A. B.: 1960, in Stellar Atmospheres (ed. by J. L. Greenstein), University of Chicago Press, Chicago.

[34] Underhill, A. B.: 1968, Ann. Rev. Astron. Astrophys. 6, 39.

[35] Hutchings, J. B.: 1968, Monthly Notices Roy. Astron. Soc. 141, 219.

[36] Hutchings, J. B.: 1968, Monthly Notices Roy. Astron. Soc. 141, 329.

[37] Feast, M. W. and Shuttleworth, M.: 1965, Monthly Notices Roy. Astron. Soc. 130, 245.

[38] Feast, M. W.: 1958, Monthly Notices Roy. Astron. Soc. 118, 618.

[39] Feast, M. W.: 1963, Monthly Notices Roy. Astron. Soc. 126, 11.

[40] Andrews, P. J.: 1968, Mem. Roy. Astron.Soc. 72, 35; and Ph.D. Thesis Cambridge, 1966.

[41] Weymann, R. (quoting H. A. Abt): 1963, Ann. Rev. Astron. Astrophys. $1,97$.

[42] Abt, H. A. and Golson, J. C.: 1966, Astrophys. J. 143, 306.

[43] Feast, M. W., Thackeray, A. D., and Wesselink, A. J.: 1960, Monthly Notices Roy. Astron. Soc. $121,337$.

[44] Peterson, D. M.: 1969, Smithsonian Astrophys. Obs. Special Report 293.

[45] Morton, D. C.: 1967, Astrophys. J. 150, 535.

[46] Underhill, A. B.: 1969, Astron. Astrophys. 1, 494.

[47] Struve, O.: 1944, Astrophys. J. 100, 189.

[48] Blaauw, A.; 1961, Bull. Astron. Inst. Neth. 15, 265.

[49] Struve, O. and Sherman, F.: 1941, Astrophys. J. 93, 84 (see also Astrophys. J. (1958) 128, 328).

[50] Henize, K. G.: 1959, Astron. J. 64, 51.

[51] Gum, C. S.: 1955, Mem. Roy. Astron. Soc. 67, 155.

[52] Westerlund, B.: 1961, Arkiv Astron. 2, 467.

[53] Henize, K. G.: 1964, Publ. Astron. Soc. Pacific 76, 385.

[54] Westerlund, B. E. and Henize, K. G.: 1967, Astrophys. J. Suppl. Ser. 14, 154.

[55] Buscombe, W. and Morris, P. M.: 1960, Monthly Notices Roy. Astron. Soc. 121, 263.

[56] Van den Bergh, S.: 1968, Astrophys. J. 151, 1191.

[57] Crampin, J. and Hoyle, F.: 1960, Monthly Notices Roy. Astron. Soc. $120,33$.

[58] Feast, M. W. and Lloyd Evans, T.: 1967, Observatory, 87, 286.

[59] Slettebak, A.: 1968, Astrophys. J. 154, 933.

[60] Feinstein, A. and Ferrer, O. E.: 1968, Publ. Astron. Soc. Pacific 80, 410.

[61] Stothers, R. and Simon, N. R.: 1968, Astrophys. J. 152, 233.

[62] Ledoux, P.: 1941, Astrophys. J.94, 537.

[63] Wilson, O. C.: 1948, Astrophys. J. 108, 201; and Schwarzschild, M. and Härm, R.: 1959, Astrophys. J.129, 637.

[64] Van Helden, R.: 1966, Bull. Astron. Inst. Netherl. 18, 367.

[65] Hutchings, J. B.: 1967, Observatory 87, 289. 
[66] Hutchings, J. B.: 1969, Non-Periodic Phenomena in Variable Stars, (ed. by L. Detre), Academic Press, Budapest p. 191.

[67] Oke, J. B.: 1954, Astrophys. J.120, 22.

[68] Thackeray, A. D.: 1937, Astrophys. J. 86, 499.

[69] Merrill, P. W.: 1947, Astrophys. J. 106, 274.

[70] Herbig, G. H.: 1969 (in press).

\section{Discussion}

Underhill: The $M_{v}$ of a star is determined by the radius of the photosphere and the brightness temperature in the $\mathrm{V}$ band. To find a spectroscopic criterion for absolute magnitude one must find lines where strengths are sensitive to the size of the photosphere and the density of gas there. This type of line has not been isolated yet in $O$ type spectra, thus one cannot separate bright $O$ f stars from less bright Of stars. Certainly the 'f' characteristics do not permit such a separation.

Viotti: In the P Cygni-like star AG Car, there is also some evidence for an outward acceleration of matter. This is a very interesting object in the same celestial region of $\eta \mathrm{Car}$, that would require a wide study in the visible and ultraviolet. 